\title{
Reducción de incidencia de neumonía asociada a ventilación mecánica post cirugía cardíaca: experiencia de 13 años de vigilancia epidemiológica en un hospital universitario
}

\author{
M. Cristina Ajenjo, Alejandra Zambrano, María I. Eugenin, Pablo Achurra, Ricardo Zalaquett, \\ Manuel J. Irarrázaval, Gabriela De la Cerda, Paula Fernández, Miriam Barañao, \\ Patricia Fuentealba, Marcela Ferrés, Patricia García, Carlos Pérez y Jaime Labarca
}

Reducing the incidence of ventilator-associated pneumonia following heart surgery: 13-year experience of epidemiologic surveillance in a teaching hospital

Objectives: To report the results of 13 years worth of epidemiologic surveillance of ventilator-associated pneumonia (VAP) following heart surgery and the main interventions applied in order to reduce VAP incidence. Methods: This is a retrospective and descriptive study of active epidemiologic surveillance of VAP. National diagnostic criteria were used. Interventions associated with a decrease in VAP incidence in adults who underwent heart surgery are described. Results: A significant and sustained reduction was observed in the rate of VAP; being 56.7 per 1,000 ventilator-days in 1998 vs 4.7 per 1,000 ventilator-days in $2010(\mathrm{p}<0.001)$. The strongest reduction was observed following 2003 (34.4 to 14.8 per 1,000 ventilator-days in 2004, p < 0.001). The interventions with greatest impact were the implementation of an early-weaning protocol, the introduction of trained nurses to perform the mechanical ventilator equipment management and the routine use of alcohol-based hand rubs. Conclusion: Epidemiologic surveillance associated with the establishment of a multifactorial intervention program applied in collaboration with the attending team, have demonstrated a significant reduction of VAP incidence after heart surgery.

Key words: Ventilator-associated pneumonia, surveillance, heart surgery

Palabras clave: Neumonía asociada a ventilación mecánica, vigilancia epidemiológica, cirugía cardíaca.

\section{Introducción}

$\mathrm{L}$

a neumonía asociada a ventilación mecánica (NAVM) es la infección más frecuente y principal complicación no-cardiológica en pacientes sometidos a cirugía cardíaca ${ }^{1}$. Su ocurrencia aumenta significativamente la morbi-mortalidad, los días de hospitalización y los costos post-operatorios asociados ${ }^{2,3}$.

La incidencia reportada de NAVM post cirugía cardíaca es de $10 \%$ en Estados Unidos de América (E.U.A. $)^{2}$ y $2,1 \%$ en España ${ }^{1}$. En las unidades de cuidados intensivos (UCI) de cirugía cardiotorácica, los Centros de Control de Enfermedades y Prevención, de Atlanta (CDC), E.U.A. reportan una mediana de 2,6 episodios de NAVM por 1.000 días de ventilación mecánica (VM) durante el período 2006-2008 ${ }^{4}$. En nuestro país, los datos de vigilancia epidemiológica del Ministerio de Salud de Chile (MINSAL) para el año 2007 muestran una mediana de 15,7 casos de NAVM por 1.000 días de VM en población general adulta ${ }^{5}$. Hasta el momento no existen datos específicos de incidencia de NAVM reportados para pacientes post-operados de cirugía cardíaca en Chile.

Desde hace varios años es un hecho bien conocido que la vigilancia epidemiológica es una estrategia fundamental y costo-efectiva para la prevención de infecciones asociadas a la atención de salud (IAAS), en particular para $\mathrm{NAVM}^{6,7}$. Nuestra institución es un centro de referencia nacional de cirugía cardíaca y tiene una larga experiencia en la prevención y control de esta complicación.

El objetivo de este estudio es comunicar los resultados de la vigilancia epidemiológica de NAVM post cirugía cardíaca y las principales intervenciones implementadas durante los últimos trece años, con el fin de reducir su incidencia.

\section{Pacientes y Métodos}

Estudio retrospectivo y descriptivo basado en los resultados de vigilancia epidemiológica de NAVM en todos los pacientes adultos sometidos a cirugía cardíaca que permanecieron 24 horas (h) o más conectados a VM con posterioridad a la cirugía, entre enero de 1998 y diciembre de 2010. El estudio se llevó a cabo en el Hospital Clínico de la Pontificia Universidad Católica de Chile (HCPUC), en Santiago de Chile. Esta institución es un complejo hospitalario universitario que actualmente consta con 481 camas, de las cuales $99(20,6 \%)$ se encuentran en
Hospital Clínico Pontificia Universidad Católica de Chile, Santiago Chile.

Comité de Prevención y Control de Infecciones Asociadas a la Atención de Salud (CPC-IAAS) (AZ, GDC, PF, $M F, P G)$.

División de Enfermedades Cardiovasculares (RZ, MJI, MB, PF). Departamento de Medicina Interna (MCA, CP, JL)

Escuela de Medicina. Pontificia Universidad Católica de Chile, Santiago Chile.

Internos de Medicina (MIE, PA).

Los autores de este trabajo declaran no tener conflictos de interés relativos al estudio.

No existió financiamiento específico para la realización de este trabajo.

Recibido: 16 de diciembre de 2011 Recepción versión definitiva: 21 de enero de 2013

Aceptado: 4 de marzo de 2013

Correspondencia a: M. Cristina Ajenjo Henríquez majenjo@puc.cl 


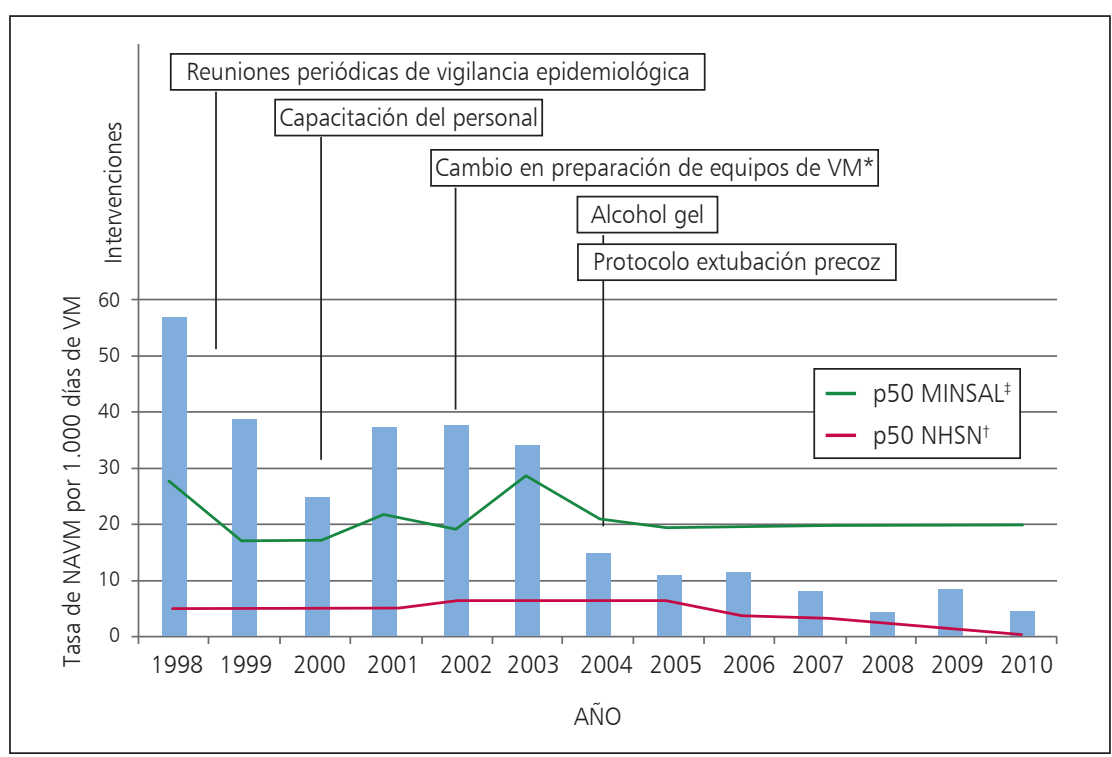

Figura 1. Incidencia de neumonía asociada a ventilación mecánica (NAVM) post cirugía cardíaca desde 1998 a 2010 y principales intervenciones realizadas a través de los años. *Cambio en preparación de equipos: incluye limpieza, esterilización y desinfección de equipos de ventilación mecánica realizada por enfermeras capacitadas en reemplazo de terapeutas respiratorios de empresa externa. ‡Percentil 50 reportado por Ministerio de Salud (MINSAL) de NAVM sin diferenciar tipo de pacientes. ${ }^{\dagger}$ Percenti 50 reportado por el National Healthcare Surveillance Network (NHSN) para UCI cardio-quirúrgica desde 2006-2010. Se utiliza reporte del National Nosocomial Infections Surveillance System (NNIS) para UCl cardiotorácica desde 1998-2005.

unidades de pacientes críticos. La vigilancia se realizó en la Recuperación de Cirugía Cardíaca y Vascular, unidad que funciona de manera independiente de los otros servicios de pacientes críticos y cuenta con ocho camas.

La vigilancia fue realizada por enfermeras entrenadas en vigilancia epidemiológica y miembros estables del Comité de Prevención y Control de Infecciones Asociadas a la Atención de Salud (CPC-IAAS) de nuestro hospital. El HCPUC cuenta con un sistema de control de calidad de la vigilancia epidemiológica basada en estudios de prevalencia anual de IAAS. Se definió NAVM en base a los criterios recomendados por el Ministerio de Salud de Chile (MINSAL) el año $1998^{8}$.

Se realizó estudio microbiológico a todos los pacientes con sospecha clínica de neumonía mediante cultivos de secreciones respiratorias. Las muestras fueron tomadas por técnica de aspirado traqueal y se analizaron como cultivo tradicional o cultivo cuantitativo, esta segunda modalidad fue incorporada desde abril de 2004. Se consideraron cultivos cuantitativos positivos todos aquellos con $\geq 10^{6}$ unidades formadoras de colonias (ufc) por ml. La identificación de los microorganismos se realizó según metodología convencional mediante pruebas bioquímicas manuales y por sistemas automatizados (Vitek2 compact $^{\mathrm{TM}}$, bioMerieux) utilizados en el laboratorio de Microbiología.
Todos los pacientes sometidos a cirugía cardíaca recibieron profilaxis antimicrobiana con cefazolina en esquema de $1 \mathrm{~g}$ intravenoso (iv) pre-operatorio y luego $1 \mathrm{~g}$ cada $8 \mathrm{~h}$ iv post-operatorio por $48 \mathrm{~h}$, pudiendo extenderse hasta el retiro de los drenajes en algunos casos. En pacientes alérgicos a $\beta$-lactámicos se utilizó vancomicina en dosis de $1 \mathrm{~g}$ iv pre-operatorio y luego 1 $\mathrm{g}$ iv cada $12 \mathrm{~h}$ post-operatorio durante $48 \mathrm{~h}$. En nuestro servicio no se utilizan corticosteroides peri-operatorios de rutina.

Las tasas de NAVM se expresaron en términos del número de casos de NAVM por cada 1.000 días de VM vigilados. No se incluyeron los pacientes conectados a VM por menos de $24 \mathrm{~h}$. Las tasas de NAVM fueron comparadas en el programa estadístico SPSS utilizando $t$-test.

\section{Resultados}

Entre enero de 1998 y diciembre de 2010 se vigilaron 5.417 cirugías cardíacas. Del total de cirugías vigiladas, 3.228 pacientes $(59,6 \%)$ se mantuvieron en VM por más de $24 \mathrm{~h}$, con un total de 7.474 días de VM vigilados. Durante el año 1998 la tasa de NAVM fue de 56,7 casos por 1.000 días de VM, siendo la tasa establecida por el MINSAL para ese año de 49,2 por 1.000 días de VM para el percentil 75 .

Se observó una reducción significativa y sostenida de la tasa de NAVM llegando a 4,7 por 1.000 días de VM durante el año 2010 ( $\mathrm{p}<0,001$ ) (Figura 1). Comparando con los datos reportados por los CDC para UCIs cardio-quirúrgicas, durante el año 2008 se obtuvo en nuestro centro una tasa de 4,25 casos por 1.000 días de VM vs 2,6 casos por 1.000 días de VM. La mayor reducción fue observada entre el año 2003 y 2004 (34,4 vs 14,8 por 1.000 días de VM, respectivamente, $\mathrm{p}<0,001)$. Desde el año 2004, las tasas de NAVM de la UCI cardio-quirúrgica se han mantenido consistentemente bajo el estándar publicado por el MINSAL para el percentil 50.

La identificación de agentes patógenos se obtuvo en $92 \%$ de las NAVM vigiladas. Los microorganismos más frecuentemente aislados fueron Enterobacteriaceae (52\%), siendo Enterobacter spp (16\%) y Klebsiella spp (14\%) los de mayor frecuencia. En segundo lugar Pseudomonas aeruginosa (19\%) y en tercer lugar Staphylococcus aureus (18,5\%) (Tabla 1).

Durante el período descrito se llevaron a cabo múltiples intervenciones para reducir la incidencia de NAVM post cirugía cardíaca. Se describe una categoría de intervenciones específicas diseñadas con el fin de disminuir la tasa de NAVM y una categoría general de conductas adoptadas de manera transversal a nivel hospitalario. 
Tabla 1. Microorganismos identificados en 148 pacientes con neumonía asociada a ventilación mecánica (NAVM)

post cirugía cardíaca entre los años 1998 y 2010

$\begin{array}{|lc|}\text { Patógeno } & \begin{array}{c}\text { Número (\%) de } \\ \text { microorganismos } \\ \text { (n }=\mathbf{1 7 8})\end{array} \\ \text { Bacterias grampositivas } & 33(18,5) \\ \text { Staphylococcus aureus } & 4(2) \\ \text { Staphylococcus coagulasa negativa } & 1(0,5) \\ \text { Otros } & \\ \text { Bacterias gramnegativas } & 93(52) \\ \text { Enterobacteriaceae } & 29(16) \\ \text { Enterobacter spp } & 25(14) \\ \text { Klebsiella pneumoniae } & 10(5,5) \\ \text { Serratia marcescens } & 29(16) \\ \text { Otras } & 40(22) \\ \text { No-fermentadoras } & 34(19) \\ \text { Pseudomonas aeruginosa } & 4(2) \\ \text { Acinetobacter baumannii } & 2(1) \\ \text { Stenotrophomonas maltophilia } & 3(1,5) \\ \text { Otras gramnegativas } & 4(2) \\ \text { Otras bacterias } & 12(8) \\ \text { Sin agente etiológico } & \end{array}$

${ }^{\dagger}$ Porcentaje (\%) en base al total de pacientes con NAVM. Hospital Clínico Pontificia Universidad Católica de Chile.

\section{Intervenciones generales:}

- A partir del año 2003 se incorporó el uso de alcohol-gel en el hospital incluyendo la Recuperación de Cirugía Cardíaca y Vascular.

- Precaución de contacto para todos los pacientes (infectados y colonizados) con microorganismos resistentes de acuerdo a la política institucional desde 2002.

- Supervisión periódica de las medidas implementadas por enfermeras del CPC-IAAS desde 1998.

\section{Intervenciones específicas:}

- A partir del año 2002 se capacitó a enfermeras de la Recuperación de Cirugía Cardíaca y Vascular para realizar la limpieza y preparación de los equipos de VM en reemplazo de terapeutas respiratorios procedentes de una empresa externa. Se incorporaron mangueras desechables, humidificadores pasivos y bolsas de ventilación estériles para cada paciente. No hubo cambios en la relación enfermeras/número de camas.

- A partir del año 2004 se implementó un protocolo de extubación precoz, evaluando activamente dentro de las primeras $24 \mathrm{~h}$ a todos los pacientes con fines de desconexión temprana de VM. Este protocolo no incluía de manera sistemática el uso de sedantes.

- Capacitaciones periódicas del personal desde el año 2000 (enfermeras, auxiliares de apoyo y personal de
Recuperación de Cirugía Cardíaca y Vascular) para el manejo de secreciones, bolsas de ventilación, nebulizaciones, oxígeno y posición semi-sentado (cabecera en posición mayor a $45^{\circ}$ ) de los pacientes en VM.

- Reuniones periódicas con el equipo médico y de enfermería de cirugía cardíaca para definir normas de vigilancia y prevención de NAVM, con el fin de revisar su cumplimiento desde 1998.

- Reactualización y revisión de normas de VM, de acuerdo a las estrategias de prevención vigente y basadas en la evidencia, disponibles en el momento de la intervención desde el año 2002.

\section{Discusión}

Este estudio confirma que es posible reducir la incidencia de NAVM posterior a cirugía cardíaca utilizando vigilancia epidemiológica activa y múltiples intervenciones a través de un período largo de tiempo. Nuestra institución, comenzó en el año 1998 la vigilancia activa en la Recuperación de Cirugía Cardíaca y Vascular con una tasa de NAVM de 56,7 por 1.000 días de VM, tasa que sobrepasaba el estándar del MINSAL recomendado como comparador (percentil 75). Durante los primeros años de vigilancia se realizaron reuniones periódicas con el equipo médico y capacitación de personal, logrando disminuir progresivamente la tasa de NAVM hasta 25 por 1.000 días de VM en el año 2000.

Desde el año 2002 en adelante se comenzaron a implementar medidas simples y de bajo costo, que respaldadas por la evidencia, han demostrado disminuir la incidencia de NAVM. La vigilancia epidemiológica y la educación del personal de salud, por ejemplo, han sido recomendadas por la Asociación Americana de Enfermedades Infecciosas como medidas generales efectivas en reducir el riesgo de NAVM y de otras infecciones asociadas a la atención de salud? 7 .

Una de las intervenciones específicas con mayor impacto en la reducción de la tasa de NAVM en nuestra institución fue el cambio del operador responsable del manejo de los equipos de VM. Existe evidencia de que el manejo adecuado de secreciones respiratorias y del circuito del VM disminuye la tasa de NAVM en pacientes graves $^{6}$. Esta medida se implementó a partir del año 2002 con su consiguiente efecto en los años siguientes, en que se observó una importante reducción de la tasa de NAVM. Respecto al equipo médico a cargo de los pacientes, es importante señalar que el grupo de Cardiocirugía no tuvo cambios en el período estudiado y los pacientes fueron operados en su gran mayoría sólo por cuatro cardiocirujanos. Existe disponibilidad de un cardiólogo estable en la unidad de Recuperación de Cirugía Cardíaca y Vascular que establece las pautas habituales y la continuidad de manejo de los pacientes. Sin embargo, existe rotación 
mensual de Becados de Cirugía Cardíaca, Vascular y Cardiología por la unidad, supervisados por los médicos estables. Considerando que esta estructura de trabajo no tuvo cambios en el período estudiado, consideramos que este factor no debe haber influido de manera importante en los resultados observados.

En nuestra experiencia, consideramos de impacto el uso de protocolos con el objetivo de disminuir el tiempo de intubación y conexión al VM; esto ha demostrado reducir la tasa de NAVM en pacientes con trauma9 ${ }^{9}$. La posición semisentada fue otra de las medidas específicas implementadas, ya que se describe en la literatura médica que esta intervención logró disminuir la tasa de NAVM desde 34 a $8 \%{ }^{10}$.

Desde el punto de vista de intervenciones generales, una de las medidas con impacto importante en la reducción de IAAS fue la introducción de productos como el alcohol-gel para la higiene de manos. Esta medida, que fue incorporada por nuestra institución el año 2003, ha demostrado mejorar la adherencia del personal hospitalario a realizar higiene de manos y disminuir la tasa de infecciones asociadas a la atención de salud (IAAS) ${ }^{11}$.

Con las medidas descritas y otras como la constante supervisión y capacitación del personal, se logró disminuir la tasa de NAVM de nuestro hospital hasta 4,7 por 1.000 días de VM en el año 2010, ubicándose por debajo del percentil 50 nacional. El MINSAL recomienda mantener la tasa de infecciones bajo el percentil 75 reportado; sin embargo, consideramos que adoptar un umbral más estricto permite un mejor control de la incidencia de IAAS. Por esta razón, la institución ha decidido adoptar como comparador el percentil 50 desde el año 2004, no sólo para NAVM, sino para todas las IAAS que se vigilan habitualmente en nuestro hospital.

En el concierto internacional existen datos específicos de la incidencia de NAVM en sub-poblaciones de pacientes, como los reportados por el National Healthcare Safety Network (NHSN) para pacientes hospitalizados en UCIs cardio-quirúrgicas ${ }^{4}$. En nuestro país se utiliza como comparador las tasas reportadas por el MINSAL que consideran la población global de adultos con NAVM, sin diferenciar el tipo de pacientes ni la unidad de origen. A conocimiento de los autores, no existe información nacional sobre la incidencia de NAVM en pacientes postoperados de cirugía cardíaca, por lo que los resultados de nuestro estudio podrían servir para establecer una referencia inicial en población chilena para este subgrupo de pacientes.

Con respecto al estudio microbiológico, los resultados obtenidos fueron similares a los publicados en la literatura científica internacional para pacientes post-operados de cirugía cardíaca ${ }^{2,12}$, identificando en nuestra serie una mayor proporción de $S$. aureus que la reportada por Kollef y cols ${ }^{2}$. En comparación con la microbiología nacional, el MINSAL reporta a Acinetobacter baumannii como el microorganismo más frecuentemente aislado, seguido por $S$. aureus y $P$. aeruginosa ${ }^{8}$. Sin embargo, nuestro grupo de pacientes no es comparable con la estadística nacional que incluye todo tipo de pacientes con NAVM y, además, nuestra institución tiene una baja endemia de infecciones por $A$. baumannii ${ }^{13}$.

Dentro de las limitaciones de este estudio podemos identificar una fuente de sesgo en el diagnóstico de NAVM. Éste se realizó en base a los criterios recomendados por el MINSAL, siendo uno de los criterios clínicos el "diagnóstico de NAVM realizado por un médico" podría presentar variabilidad entre cada profesional, no siempre es reproducible y se trata de una variable no estandarizada. El resto de los criterios clínico-radiográficos son, sin embargo, observaciones objetivas y estandarizadas. La mayoría de los estudios internacionales sobre NAVM realizan el diagnóstico en base a los criterios propuestos por los CDC, los que no consideran la variable previamente mencionada y comparten la dificultad de interpretación de los infiltrados pulmonares que son frecuentes en los pacientes post operados de cirugía cardíaca ${ }^{14}$.

Otra limitación es que este estudio no considera de manera diferenciada las variables propias del paciente (por ej: edad, co-morbilidades, capacidad funcional), ni tampoco las características de la cirugía (por ej: cirugía de emergencia, tipo de cirugía cardíaca, tiempo operatorio, tiempo en bypass y clamping aórtico). Muchas de estas variables han sido identificadas como factores de riesgo para el desarrollo de NAVM post cirugía cardíaca en la literatura médica ${ }^{12}$. Así, no es posible asegurar que los pacientes operados a través de los años sean comparables. Sin embargo, por las características propias de nuestra institución y la complejidad creciente de los pacientes atendidos, postulamos que este factor probablemente influye poco en los resultados observados, particularmente por el número de pacientes y el tiempo prolongado de seguimiento del estudio.

Por el carácter retrospectivo del estudio, no fue posible incluir en los resultados datos respecto a mortalidad y a evolución de los pacientes que presentaron NAVM post cirugía cardíaca. Así mismo, no fue posible obtener información microbiológica más detallada respecto a la frecuencia de microorganismos multi-resistentes como agentes causales de NAVM para todos los años estudiados.

Es importante destacar que varias de las intervenciones para reducir la incidencia de NAVM post cirugía cardíaca fueron realizadas de manera simultánea y aditiva en el tiempo, lo que no permite establecer el impacto de cada una de ellas por separado. Además, por ser un estudio retrospectivo, no fue posible precisar en todas ellas el momento exacto de implementación de la intervención en el tiempo, sólo establecer el año en que introdujeron los cambios. Tampoco fue posible cuantificar de manera 
objetiva el grado de cumplimiento de todas las intervenciones durante todos los años de seguimiento.

Una de las principales fortalezas de este estudio es el largo tiempo de seguimiento en el que se llevó a cabo. Esto nos permite identificar una tendencia estadísticamente significativa y progresiva en el tiempo, lo que constituye un aporte al manejo de esta complicación de alto impacto en pacientes con cirugía cardíaca.

En la actualidad, una de las estrategias más aceptadas en prevención de NAVM es la incorporación de "bundles" ${ }^{15,16}$ o paquetes de medidas aplicadas en conjunto para prevenir IAAS. Se trata de una intervención compleja que consume tiempo y requiere de importante colaboración del equipo de UCI. En estudios recientes la incorporación de cinco medidas tales como: a) sólo cambiar los circuitos de ventilación cuando está clínicamente indicado; b) incorporación de protocolos de sedación; c) estricta higiene de manos con alcohol-gel antes de manipular los circuitos de ventilación; d) aseo de la cavidad oral con clorhexidina cada 8 h y e) control de la presión del cuff del tubo endotraqueal, han demostrado una reducción significativa de las tasas de $\mathrm{NAVM}^{17}$. Nuestra institución está evaluando incorporar bundles para prevención de NAVM, porque si bien se ha avanzado mucho y nuestras tasas de NAVM son bajas, creemos que aún queda mucho por hacer en este tema. Sin embargo, una de las limitaciones encontradas en la aplicación de bundles ha sido la baja adherencia a las medidas, no superior a $30 \%{ }^{17}$.

En base a los resultados de este estudio, podemos concluir que el trabajo conjunto entre los servicios clínicos y el equipo de control de infecciones, la continuidad de vigilancia epidemiológica activa y la implementación de intervenciones costo-efectivas, pueden reducir significativamente la incidencia de NAVM en pacientes post-operados de cirugía cardíaca.

Una de las virtudes de la vigilancia epidemiológica activa es la promoción de un constante perfeccionamiento dentro del personal de salud. Asimismo, al realizar vigilancia seriada, podemos identificar de manera temprana situaciones en las cuales las tasas de NAVM se escapan al objetivo propuesto por cada servicio. Creemos que es muy importante continuar reportando resultados e investigando en esta área, lo cual es crítico para seguir mejorando la calidad de la atención y la seguridad de nuestros pacientes.

Agradecimientos. Los autores agradecen sinceramente el trabajo realizado por las enfermeras del Comité de Prevención y Control de Infecciones Asociadas a Atención de Salud (CPC-IAAS) y el personal de Recuperación de Cirugía Cardíaca y Vascular del Hospital Clínico de la Pontificia Universidad Católica de Chile.

\section{Resumen}

Objetivos: Comunicar los resultados de 13 años de vigilancia epidemiológica de neumonía asociada a ventilación mecánica (NAVM) post cirugía cardíaca y las principales intervenciones implementadas para reducir su incidencia. Metodología: Estudio retrospectivo, descriptivo, de vigilancia epidemiológica activa de NAVM utilizando los criterios del Ministerio de Salud (MINSAL) y de las intervenciones asociadas con una disminución de la tasa de NAVM en adultos operados de cirugía cardíaca. Resultados: Se observó una reducción significativa y sostenida de la tasa de NAVM, siendo 56,7 por 1.000 días de ventilación mecánica (VM) en 1998 vs 4,7 por 1.000 días de $\mathrm{VM}$ en 2010 ( $\mathrm{p}<0,001)$. La mayor reducción fue observada a partir de 2003 (desde 34,4 a 14,8 por 1.000 días de VM en 2004, p < 0,001). Las intervenciones con mayor impacto fueron la implementación de un protocolo de extubación precoz, la incorporación de enfermeras capacitadas en el manejo de los equipos de VM y el uso rutinario de alcohol gel. Conclusión: La vigilancia epidemiológica asociada a un programa de intervención multifactorial aplicado en conjunto con el equipo tratante permitió reducir significativamente la incidencia de NAVM post cirugía cardíaca.

\section{Referencias bibliográficas}

1.- Hortal J, Muñoz P, Cuerpo G, Litvan H, Rosseel P, Bouza E. Ventilator-associated pneumonia in patients undergoing major heart surgery: an incidence study in Europe. Crit Care 2009; 13: 1-10.

2.- Kollef M H, Sharpless L, Vlasnik J, Pasque C, Murphy D, Fraser V. The impact of nosocomial infections on patient outcomes following cardiac surgery. Chest 1997; 112: 666-75.

3.- Welsby I J, Bennett-Guerrero E, Atwell D, White W, Newman M, Smith P, et al. The association of complication type with mortality and prolonged stay after cardiac surgery with cardiopulmonary bypass. Anesth Analg 2002; 94: 1072-8.

4.- Edwards J R, Peterson K D, Mu Y, Banerjee S, Allen-Bridson K, Morrell G, et al. National Healthcare Safety Network (NHSN) report: Data summary for 2006 through 2008. Am J Infect Control 2009; 37: 783-805.

5.- Bustamante R, Espínola V. Vigilancia epidemiológica de infecciones intrahospitalarias, 2007. www.minsal.cl.

6.- Coffin S E, Klompas M, Classen D, Arias K M, Podgorny K, Anderson D, et al. Strategies to prevent ventilator-associated pneumonia in acute care hospitals. Infect Control Hosp
Epidemiol. 2008; 29: S31-40.

7.- Tablan O C, Anderson L J, Besser R, Bridges C, Hajjeh R. Guidelines for preventing health-care associated pneumonia, 2003: Recommendations of the CDC and Healthcare Infection Control Practices Advisory Committee . Respir Care 2004; 49: 926-39.

8.- Otaíza F, Brenner P. Sistema de vigilancia de infecciones intrahospitalarias, 1998. Anexo 1. Programa de IIH de la División de Salud de las Personas del Ministerio de Salud de Chile. http://www.minsal.gob.cl/portal/url/item/8a3316 13f071f5e7e04001011e010f96.pdf

9.- Marelich GP, Murin S, Battistella F, Inciardi J, Vierra T, Roby M. Protocol weaning of 
mechanical ventilation in medical and surgical patients by respiratory care practitioners and nurses: effect on weaning time and incidence of ventilator-associated pneumonia. Chest 2000; 118: 459-67.

10.- Drakulovic M B, Torres A, Bauer T T, Nicolas J M, Nogué S, Ferrer M. Supine body position as a risk factor for nosocomial pneumonia in mechanically ventilated patients: a randomised trial. Lancet 1999; 354: 1851-8.

11.- Pittet D, Hugonnet S, Harbarth S, Mourouga P, Sauvan V, Touveneau S, et al. Effectiveness of a hospital-wide programme to improve compliance with hand hygiene. Infection Control Programme. Lancet 2000; 356: 1307-12.
12.- Bouza E, Pérez A, Muñoz P, Jesús Pérez M, Rincón C, Sánchez C, et al. Ventilatorassociated pneumonia after heart surgery: a prospective analysis and the value of surveillance Crit Care Med 2003; 31: 1964-70.

13.- Labarca J, Rivera G, Castillo C, García P. Caracterización epidemiológica de Acinetobacter baumannii resistente a carbapenémicos aislados en un hospital universitario: rol de pacientes transferidos desde otros hospitales. CO-29. XXVIII Congreso Chileno de Infectología, La Serena, Chile 2011. Rev Chilena Infectol 2011; 28 (S2): 124.

14.- Garner J S, Jarvis W R, Emori T G, Horan T C, Hughes J M. CDC definitions for nosocomial infections, 1988. Am J Infect Control 1988; 16:
$128-40$.

15.- Cruden E, Boyce C, Woodman H, Bray B. An evaluation of the impact of ventilator care bundle. Nurs Crit Care 2005; 10: 242-6.

16.- Rello J, Lode H, Cornaglia G, Masterton R on behalf of the VAP Care Bundles Contributors. A European care bundle for prevention of ventilator-associated pneumonia. Intensive Care Med 2010; 36: 773-80.

17.- Rello J, Afonso E, Lisboa T, Ricart M, Balsera B, Rovira A, et al. FADO Project Investigators. A care bundle approach for prevention of ventilator-associated pneumonia. Clin Microbiol Infect 2012 Feb 9. doi: 10.1111/j.1469-0691.2012.03808.x 